\title{
Critical Indices and Self-Similar Power Transform
}

\author{
Simon Gluzman
}

Citation: Gluzman, S. Critical Indices and Self-Similar Power Transform. Axioms 2021, 10, 162. https://doi.org/10.3390/ axioms10030162

Academic Editor: Davron Aslonqulovich Juraev

Received: 16 June 2021

Accepted: 23 July 2021

Published: 26 July 2021

Publisher's Note: MDPI stays neutral with regard to jurisdictional claims in published maps and institutional affiliations.

Copyright: (C) 2021 by the author. Licensee MDPI, Basel, Switzerland. This article is an open access article distributed under the terms and conditions of the Creative Commons Attribution (CC BY) license (https:// creativecommons.org/licenses/by/ $4.0 /)$.
Materialica and Research Group, Bathurst St. 3000, Apt. 606, Toronto, ON M6B 3B4, Canada; simongluzmannew@gmail.com

Abstract: "Odd" factor approximants of the special form suggested by Gluzman and Yukalov (J. Math. Chem. 2006, 39, 47) are amenable to optimization by power transformation and can be successfully applied to critical phenomena. The approach is based on the idea that the critical index by itself should be optimized through the parameters of power transform to be calculated from the minimal sensitivity (derivative) optimization condition. The critical index is a product of the algebraic self-similar renormalization which contributes to the expressions the set of control parameters typical to the algebraic self-similar renormalization, and of the power transform which corrects them even further. The parameter of power transformation is, in a nutshell, the multiplier connecting the critical exponent and the correction-to-scaling exponent. We mostly study the minimal model of critical phenomena based on expansions with only two coefficients and critical points. The optimization appears to bring quite accurate, uniquely defined results given by simple formulas. Many important cases of critical phenomena are covered by the simple formula. For the longer series, the optimization condition possesses multiple solutions, and additional constraints should be applied. In particular, we constrain the sought solution by requiring it to be the best in prediction of the coefficients not employed in its construction. In principle, the error/measure of such prediction can be optimized by itself, with respect to the parameter of power transform. Methods of calculation based on optimized power-transformed factors are applied and results presented for critical indices of several key models of conductivity and viscosity of random media, swelling of polymers, permeability in two-dimensional channels. Several quantum mechanical problems are discussed as well.

Keywords: minimal sensitivity; optimization; power transform; critical index

\section{Introduction}

Often, the limit-problems are characterized by power laws. Accurate analytical formulae for deterministic and random systems, such as composites, suspensions, and porous media, can be derived by employing approximants, when the low-concentration series are supplemented with information on the high-concentration regime near divergence points signifying the physical percolation effects [1,2].

Sometimes, the results can be improved by simple transformation to the divergent quantities. It is often assumed that even a naive inverse transformation of the original truncated series could be accomplished. Generally, one can introduce the so-called power transform defined in [3]. Below, we consider application of power transform to calculation of the critical properties and of the critical index in particular. It is possible to find the critical index as an explicit function of the parameters defining the power transformation. Instead of optimization of critical amplitudes as in [3-7], we express and optimize the critical index directly. The critical index is obtained then by finding some optimal power transformation, demanding the critical index to be minimally sensitive to the parameters of power transform.

Indeed, as we are interested in the critical index, it seems more natural to optimize the index directly, rather than to try to find from optimizing some other quantities, even the critical amplitudes. Furthermore, as the critical indices are not sensitive to the fine details 
of interactions, or to the parameters imposed in the course of optimization, we apply rather generic optimization conditions to rather short expansions.

Various power transformations are known in statistics. Most notable are the Tukey's ladder of powers [8] and Box-Cox transformation [9]. The Box-Cox transformation is nothing else but the celebrated replica-trick. It is intimately related to the concept of highly optimized tolerance (HOT) [10]. HOT is viewed as the mechanism behind the power laws.

Formally, we study the behaviour of a real function $\phi(x)$ of a real variable $x \in[0, \infty)$. This function is typically defined by a complicated problem that does not allow for an explicit knowledge of the functional form. However, one can attempt to develop some kind of perturbation theory. The perturbation theory yields truncated asymptotic expansions representing the function

$$
\phi(x) \simeq \phi_{k}(x) \quad(x \rightarrow 0)
$$

at a small variable $x \rightarrow 0$, with $k=0,1, \ldots$ being perturbation order. The perturbative series of $k$ th order can be written as an truncated expansion in powers of $x$ as

$$
\phi_{k}(x)=a_{0}+\sum_{n=1}^{k} a_{n} x^{n} .
$$

Unless otherwise stated, $a_{0}=1$ is chosen. The series (2) will be subject to renormalization. The most difficult region for approximating is that of the large variable. Our main interest here will be to find the large-variable behaviour of the function, where its asymptotic form is expected to be

$$
\phi(x) \simeq B x^{\beta} \quad(x \rightarrow \infty) .
$$

The constant $B$ will be called the critical amplitude and the power $\beta$ is the critical exponent.

We have to transform the truncated series into convergent expressions with the asymptotic behavior (3). We are going to calculate $\beta$ first, and $B$ second, by employing the technique of power transforms applied to factor approximants [3].

One can consider the index $k$ as the discrete time. Then, the truncated series (2) corresponds to the points of the trajectory of the dynamical system. The velocity which governs the passage from one point to another is approximated from the available truncated series. Finding the stable fixed points for such a dynamical system means to be able to find the sum (1) as $k \rightarrow \infty$.

In the vicinity of the fixed point, the functional self-similarity relation between the consecutive functional approximations can be employed constructively. Moreover, due to the self-similarity relation, one can look for the fixed point representing the sought sum in the analytical form, e.g., the power-transformed factor approximant can be considered as such a representation [3]. For such approximants, one can analytically express the critical index and amplitude, and optimize the critical index with respect to the parameters of power transform. To define the power transform in full, we have to apply additional, optimization conditions of rather general nature. More specifically, the program could be accomplished by means of the power-transformed "odd" factor approximants [3]. Furthermore, the conditions on fixed points using power transform parameters as control parameters take particularly simple form and optimization leads to some polynomial-type equations, relatively easy to solve numerically.

The general idea of application of various optimal conditions in the space of approximations is due to V.I. Yukalov [11,12]. For the particular example, minimal difference condition was put forward in [13]. Minimal sensitivity condition was exploited in [14]. Kleinert's variational-perturbation method also employs minimal derivative conditions [15]. However, the method is very difficult to adapt to the purpose of finding an analytical expression for the sought quantity.

The method of coherent anomaly was suggested and developed by M. Suzuki $[16,17]$. He applied the ideas of the renormalization group, but specifically considered it in the 
space of approximations. The physical quantities are considered with respect to the approximation parameter, discrete in most cases, just as in the general approach briefly discussed above. The method allows to estimate critical indices but does not bring the concrete approximant whatsoever. Absence of control parameters makes it necessary to consider high-order approximations to the sought quantity in order to achieve better results.

Various self-similar techniques were applied to calculations with short series $[5,6,18]$, but they all appear to be designed for providing lower and upper bounds for the index. Such methods combine at least two separate methods and are applied through optimizing the expressions for critical amplitudes [2,5]. Such an indirect approach to the critical indices computations can be remedied by reformulating it for the direct optimization of the expressions for critical indices. However, to accomplish the task, it turned out to be necessary to extend the class of approximations in order to be able to select possible stable solutions within the bounds found by other methodologies.

Because of the limited information supplied by the short series, the problem of critical properties should be attacked by various methods, under various assumptions [2] The method considered in the current paper does have some unique qualities, such as incorporating both critical and sub-critical indices simultaneously, while the optimization procedure is applied directly to the critical index. It appears to give more stable performance than all other methods based on finding the index as a control parameter from the critical amplitude optimization.

\section{Power Transform and Minimal Sensitivity Condition for the Index}

It is possible to improve the quality of approximants by employing power transforms [3]. To this end, we defined the power transform of the reduced expansion (2) as

$$
P_{k}(x, m) \equiv \phi_{k}^{m}(x) .
$$

In turn, the power transform (4) can be expanded in powers of $x$ giving

$$
P_{k}(x, m) \simeq \sum_{n=0}^{k} b_{n}(m) x^{n}
$$

After the self-similar renormalization of the expansion (5) is accomplished by means of, say, factor approximants, we arrive at an approximant $\mathcal{P}_{k}^{*}(x, m)$. Then, we accomplish the inverse transformation

$$
F_{k}(x, m)=\left[\mathcal{P}_{k}^{*}(x, m)\right]^{1 / m} .
$$

Thus, in distinction with the statistics, we rigidly demand the existence of a direct and inverse transformation, eradicating an uncertainty in the matter of interpretation existing in the statistical applications $[8,9]$.

The powers $m_{k}=m_{k}(x)$ are defined by the variational condition

$$
\frac{\partial F_{k}(x, m)}{\partial m}=0 .
$$

Finally, the corresponding approximation for the sought function is given by

$$
\phi_{k}^{*}(x)=F_{k}\left(x, m_{k}\right) \text {. }
$$

Suppose that after all transformations performed explicitly one can find an explicit expression for the critical index $\beta_{k}(m)$, in $k$ th order. When we are interested in the power law appearing in the large-variable limit and the critical index in particular, the condition (7) reduces to the requirement that the critical index should not depend on the parameter of transformation expressed in the following form:

$$
\frac{\partial \beta_{k}(m)}{\partial m}=0
$$


The differentiation of the critical index leads to the minimal sensitivity condition on the parameter of transformation $m$.

Although equation (9) is quite intuitive, it can be explained following the idea put forward in [7]. Assume that we found the expression for the critical index

$$
\beta(m) \approx \beta_{k}(m),
$$

in $k$ th approximation. Correspondingly, we can find the critical amplitude for the $k$ th approximation $B_{k}(m) \approx B(m)$. Here, we should also recognize that $B(m)=B(\beta(m))$. As at large $x$

$$
\phi_{k}^{*}(x) \simeq B x^{\beta}
$$

the differentiation (7) can be performed, and the following expression obtained for large $x$,

$$
\frac{\partial \phi_{k}^{*}(x)}{\partial m} \simeq x^{\beta}\left(\frac{\partial B}{\partial \beta}+B \ln x\right) \frac{\partial \beta}{\partial m} .
$$

Thus, as long as we are interested in large $x$ critical properties, in order to make the approximation $F_{k}(x, m)$ minimally sensitive to the transformation parameters it is sufficient to satisfy the equation (9).

To put the meaning into the formulas one is bound to select the self-similar approximants. Following the work in [3], we consider the $k$ th order "odd" self-similar factor approximant

$$
\mathcal{P}_{k}^{*}(x)=1+\mathcal{A} x \prod_{i=1}^{N_{k}-1}\left(1+\mathcal{A}_{i} x\right)^{s_{i}}, \quad N_{k}=\frac{1+k}{2}, k=3,5, \ldots
$$

The parameters $\mathcal{A}, \mathcal{A}_{i}$ and $s_{i}$ have to be defined from an odd number of $k$ conditions. The necessary number of conditions typically is extracted from the conditions of asymptotic equivalence with the truncated series.

In our study, we insist only that the total number of conditions employed should be equal to $k$. The approximant can be considered as "odd" [3], while factor approximants of "even" type, recently considered as subject of optimization [6], are simply not amenable to the optimization by power transform. They should be approached by some other optimization techniques developed previously in $[6,7]$.

With $k=3$ we simply have

$$
\mathcal{P}_{3}^{*}(x)=1+\mathcal{A} x\left(1+\mathcal{A}_{1} x\right)^{s_{1}} .
$$

As $x \rightarrow \infty$

$$
F_{3}^{*}(x, m) \simeq\left(\mathcal{A}(m)\left(\mathcal{A}_{1}(m)\right)^{s_{1}(m)}\right)^{1 / m} x^{\left(1+s_{1}(m)\right) / m} .
$$

Minding that $\mathcal{A}, \mathcal{A}_{1}$ and $s_{1}$ are functions of $m$, the critical index can be found

$$
\beta \approx \beta_{3}(m)=\frac{1+s_{1}(m)}{m}
$$

but only as the function of parameter $m$. It has to be found from the simplified minimal sensitivity condition

$$
\frac{\partial \beta_{3}(m)}{\partial m}=0
$$

After the particular value of $m_{3} \equiv m^{*}$ is found, one can find the the critical index from the formula (13), and the critical amplitude

$$
B \approx B_{3}\left(m^{*}\right)=\left(\mathcal{A}\left(m^{*}\right) \mathcal{A}_{1}\left(m^{*}\right)^{s_{1}\left(m^{*}\right)}\right)^{1 / m^{*}}
$$


The form of approximation expressed by the formula (12) is common for all selfsimilar approximants known to us, such as roots, factors, additive approximants, continued roots $[3,19,20]$, i.e., it is not sensitive at all to the assumptions made while constructing the approximations in higher-orders, and includes the basic, single step of the algebraic self-similar transformation, as explained in [20,21].

For $k=5$ we have

$$
\mathcal{P}_{5}^{*}(x)=1+\mathcal{A} x\left(1+\mathcal{A}_{1} x\right)^{s_{1}}\left(1+\mathcal{A}_{2} x\right)^{s_{2}} .
$$

As $x \rightarrow \infty$

$$
F_{5}^{*}(x, m) \simeq\left(\mathcal{A}(m)\left(\mathcal{A}_{1}(m)\right)^{s_{1}\left(m^{*}\right)}\left(\mathcal{A}_{2}(m)\right)^{s_{2}(m)}\right)^{1 / m} x^{\left(1+s_{1}(m)+s_{2}(m)\right) / m} .
$$

With

$$
\beta \approx \beta_{5}(m)=\frac{1+s_{1}(m)+s_{2}(m)}{m},
$$

the parameter $m_{5} \equiv m^{*}$ has to be found from the minimal sensitivity condition

$$
\frac{\partial \beta_{5}(m)}{\partial m}=0
$$

One can also find the critical amplitude

$$
B \approx B_{5}\left(m^{*}\right)=\left(\mathcal{A}\left(m^{*}\right)\left(\mathcal{A}_{1}\left(m^{*}\right)\right)^{s_{1}\left(m^{*}\right)}\left(\mathcal{A}_{2}\left(m^{*}\right)\right)^{s_{2}\left(m^{*}\right)}\right)^{1 / m^{*}} .
$$

In order to simplify formulas without modifying the expressions for critical index and minimal sensitivity equations, we are going to set $\mathcal{A}_{2}(m)=1$ (see, e.g., in [7]). However, imposing such conditions in lower-order would not bring a solution to the optimization problem.

The form of the expressions for critical induces (13) and (15), suggests that in special case when the optimal value is found as $m^{*}=0$ (or close), one can start, or include into consideration at par with other approximations, some logarithmic-type approximations as discussed, e.g., in [22]. As $m=1$, the formulas (13) and (15) will correspond to the results for the critical index originating only from the factor approximants (11) per se.

In the general case of arbitrary $m$, the critical index is a product of the algebraic transformation which contributes the set of control parameters $s_{i}$, and of the power transform which corrects them in two ways. First, $s_{i}$ are getting "dressed" becoming dependent on the parameter of power transform $m$ and, second, they are divided by $m$.

The parameter of power transformation $m$ has a simple meaning of the multiplier connecting the critical exponent with the correction-to-scaling exponent, to be explained below in the Section 3.1. Both the power transform and minimal sensitivity condition are of a non-perturbative origin, based on generic ideas on improving the quality of perturbative calculations. The expression (15) can be with ease generalized to the $k$ th order,

$$
\beta \approx \beta_{k}(m)=\frac{1+s_{1}(m)+s_{2}(m)+\ldots s_{k}(m)}{m} .
$$

\section{Critical Point at Infinity}

As long as we are concerned with the low-order calculations, it makes sense to simplify the problem even further. In order to analytically solve the minimal problem, with only two non-trivial coefficients, $a_{1}$ and $a_{2}$, let us impose an additional condition on the amplitudes, namely,

$$
\mathcal{A}_{1}=\mathcal{A} .
$$

This condition does not directly involve the shape of the minimization condition imposed exclusively on the critical index. Imposing the constraint also allows to greatly 
simplify calculations and obtain a unique solution for the optimization problem in lowest non-trivial order. Besides, it also guarantees the existence of the (approximate) solution by guaranteeing positive amplitudes.

From the asymptotic equivalence with the truncated series, we find

$$
\mathcal{A}_{1}(m)=\mathcal{A}(m)=a_{1} m, \quad s_{1}(m)=\frac{a_{1}^{2} m-a_{1}^{2}+2 a_{2}}{2 a_{1}^{2} m} .
$$

From the optimization condition, we find the optimal control parameter

$$
m^{*}=\frac{2\left(a_{1}^{2}-2 a_{2}\right)}{3 a_{1}^{2}} .
$$

It leads to the critical index

$$
\beta \approx \beta_{3}=\frac{9 a_{1}^{2}}{8\left(a_{1}^{2}-2 a_{2}\right)},
$$

and critical amplitude

$$
B \approx B_{3}=\left(\frac{2}{3}\right)^{\frac{9 a_{1}{ }^{2}}{8\left(a_{1}{ }^{2}-2 a_{2}\right)}}\left(a_{1}-\frac{2 a_{2}}{a_{1}}\right)^{\frac{9 a_{1}{ }^{2}}{8\left(a_{1}{ }^{2}-2 a_{2}\right)}} .
$$

See Section 3.4 for more general formulas (17) in the case of $\mathcal{A}_{1}(m) \neq \mathcal{A}(m)$.

For longer series, the optimization condition possesses multiple solutions and additional constraints should be applied. In particular, we require that the chosen solution should be best in prediction of the coefficients $a_{k}$ not employed in its construction.

The optimization condition leads to the equation on high-order polynomials in the parameter $m$, so that an exact solution cannot be found. On the other hand, the class of solutions is significantly broadened because the complex-conjugate roots are now allowed. In some important examples presented below they appear to give the best results when the constraints are imposed. In principle, the error/measure of such prediction can be optimized by itself, with respect to the parameter of power transform.

\subsection{Swelling of Polymers}

An important characteristic of polymer chains is their swelling factor. It is just the ratio of the mean-square end-to-end distance of the chain, with interactions between its segments, to the value of the mean-square end-to-end distance of the chain, without such interactions.

Two-dimensional polymers are often met in chemistry and biology. For such polymers, perturbation theory with respect to weak interactions can be developed [23,24]. It can be reduced to a truncated series in a single dimensionless interaction parameter $g$. For the the expansion factor $Y(g)$, it gives

$$
\mathrm{Y}(g) \simeq 1+\frac{1}{2} g-0.12154525 g^{2}+0.02663136 g^{3}-0.13223603 g^{4},
$$

as $g \rightarrow 0$.

In the strong-interaction limit $[25,26]$, one expects the power law behavior

$$
Y(g) \simeq B g^{\beta} \quad(g \rightarrow \infty),
$$

with the critical exponent $\beta=1 / 2$. One also considers the critical index $v \equiv \frac{1}{2}(1+\beta)$, which gives $v=0.75$.

Application of the formulas presented above gives $m^{*}=1.31491, \beta=\beta_{3}=0.570382$, $B=B_{3}=0.787252$. Correspondingly, $v \approx 0.785191$, well within the bounds $v=0.77525 \pm$ 0.021747 calculated in [2]. 
In the case of a three-dimensional polymer coil, perturbation theory [23] for the swelling factor leads to series of the same type as (21), but with the coefficients

$$
\begin{gathered}
a_{1}=\frac{4}{3}, \quad a_{2}=-2.075385396, \quad a_{3}=6.296879676, \\
a_{4}=-25.05725072, \quad a_{5}=116.134785, \quad a_{6}=-594.71663 .
\end{gathered}
$$

The strong-coupling limit of the swelling factor $Y(g)$ can be found from [24], and

$$
Y(g) \simeq 1.531 g^{0.3544} \quad(g \rightarrow \infty),
$$

By applying our approach, we obtain $m^{*}=2.22321, \beta=\beta_{3}=0.337351, B=B_{3}=$ 1.44279 and the critical index $v \approx 0.584338$. Complete swelling factor looks rather simple,

$$
Y(g)=\phi_{3}^{*}(g)=\left(1+\frac{2.96427 g}{(2.96427 g+1)^{0.25}}\right)^{0.449801} .
$$

The result for the critical index is located within the bounds $v=0.5814 \pm 0.006$ found earlier in [5], based on the same input form the truncated series.

One may expect that the higher-order terms will have some effect on the results. There are multiple solutions to the minimal sensitivity equation. The best solution to the minimal sensitivity problem corresponds to

$$
m_{1}^{*}=2.31467
$$

The corresponding approximant reads as follows,

$$
Y(g)=\prec_{5}^{*}(g)=\left(1+\frac{3.08622 g}{(g+1)^{0.0869825}(6.21143 g+1)^{0.0954871}}\right)^{0.432028} .
$$

Asymptotically, at large $g$

$$
\phi_{5}^{*}(g) \simeq B g^{\beta},
$$

and the critical indices $\beta=0.353196, v=0.588299$, and the critical amplitude $B=1.50912$. Compared to all other solutions to the minimal sensitivity condition, formula (24) gives the smallest average error in estimation of the 5th and 6th order coefficients in the expansion. The results fit within the bounds marked by the numerical result $v=0.5886$ [24], and by the numerical result $v=0.5877$ of [27], or even by a slightly lower value of 0.5876 obtained in [28].

Intriguingly, the "odd" factor approximants contain also the correction-to-scaling exponent $\Delta$ defined in $[2,24,29]$, as follows:

$$
\mathrm{Y}(g) \simeq B g^{\beta}\left(1+\frac{B_{1}}{B} g^{-\Delta}\right)(g \rightarrow \infty) .
$$

After extraction of the critical behavior, and by rewriting the transformed factor approximants for $k=3,5$, one can see that in $k$ th order

$$
\Delta_{k}=\beta_{k}(m) m .
$$

Note that the technique of optimization from [6], which also dwells on the factor approximants, always brings $\Delta \equiv 1$. The case of $k=3$ is also qualitative, always bringing $\Delta=0.75$.

However, for $k=5$, we simply calculate $\Delta_{5} \approx 0.82$, in a good agreement with the numerical estimate $\Delta=0.93$ from [24]. Thus the parameter $m$ of the power transformation is the multiplier connecting the two exponents. The connection is of general nature and not limited to the case of polymers. Analysis of available literature does confirm that in all 
cases considered in this section, such simultaneous estimation of the two indices works reasonably well.

\subsection{Schwinger Model}

The Schwinger model $[30,31]$ represents the Euclidean quantum electrodynamics interacting with a Dirac fermion field, defined on a lattice in $(1+1)$ dimensions. The model reflects confinement, chiral symmetry breaking, and charge shielding, sharing therefore the key properties with quantum chromodynamics. The ground state of the model, given as a function of the dimensionless variable $x=m / g$. Here, $m$ stands for electron mass and $g$ is the coupling parameter. It also has the dimension of mass. The energy $E=M-2 m$, corresponds to a vector boson of mass $M(x)$. ing form:

The expansion at small- $x$ for the ground-state energy [32-35] is known in the follow-

$$
E(x) \simeq 0.5642-0.219 x+0.1907 x^{2} \quad(x \rightarrow 0) .
$$

In the complementary, large- $x$ limit [35-38], there is a power law,

$$
E(x) \simeq B x^{\beta}+O\left(x^{-1}\right) \quad(x \rightarrow \infty),
$$

with $B=0.6418, \beta=-1 / 3$.

To standardize calculations, let us first normalize the expansion (26) to unity at $x=0$. Elementary calculations according to the formulas presented above, give $m^{*}=-2.32446$, $\beta \approx \beta_{3}=-0.322656, B_{3}=1.03374$. Restoring the original units, we find the critical amplitude $B=0.583237$. The result for the index fits within the bounds $\beta=-0.311 \pm 0.2$ found in [5].

\subsection{Harmonium}

An $N$-electron harmonium atom is described by the Hamiltonian

$$
\hat{H}=\frac{1}{2} \sum_{i=1}^{N}\left(-\nabla_{i}^{2}+\omega^{2} r_{i}^{2}\right)+\frac{1}{2} \sum_{i \neq j}^{N} \frac{1}{r_{i j}},
$$

where dimensionless variables are used, $\frac{\omega^{2}}{2}$ stands for the harmonic oscillator force constant [39], and $r_{i} \equiv\left|\mathbf{r}_{\mathbf{i}}\right|, r_{i j} \equiv\left|\mathbf{r}_{\mathbf{i}}-\mathbf{r}_{\mathbf{j}}\right|$.

Following Cioslowski [39], we consider a two-electron harmonium atom with $N=2$. The ground-state energy for a rigid potential diverges as the power law [39] at large $\omega$,

$$
E(\omega)=3 \omega+O\left(\omega^{1 / 2}\right) \quad(\omega \rightarrow \infty) .
$$

At a shallow harmonic potential, the energy can be expanded [39] in powers of $\omega$ giving in low orders the following truncation:

$$
E(\omega) \simeq 1.19055 \omega^{2 / 3}+2.36603 \omega+0.122492 \omega^{4 / 3} \quad(\omega \rightarrow 0) .
$$

By introducing the new variable $x \equiv \omega^{1 / 3}$, Equation (30) could be reduced to

$$
E\left(x^{3}\right) \simeq 1.19055 x^{2}\left(1+1.98734 x+0.102887 x^{2}\right) \quad(x \rightarrow 0) .
$$

Applying our method to the expression within brackets, we find $m^{*}=0.631933$, $\beta_{3}=1.18684, B_{3}=1.31048$, and reconstruct the large $\omega$ behaviour

$$
E(\omega) \simeq B \omega^{\beta} \quad(\omega \rightarrow \infty)
$$


with $B=3.10063, \beta=1.06228$. The error of $6 \%$ for the critical index should be considered as quite satisfactory for calculations with such short truncation as (30). The critical index is well within the bounds $\beta=1.049 \pm 0.031$ found in [5].

\subsection{Nonlinear Schrödinger Equation}

The following nonlinear Hamiltonian

$$
\hat{H}_{N L S}=-\frac{1}{2} \frac{d^{2}}{d x^{2}}+\frac{1}{2} x^{2}+g|\psi|^{2},
$$

defines the one-dimensional stationary nonlinear Schrödinger equation for the wave function $\psi$ of the Bose-condensed atoms in a harmonic trap. Here, $g$ is a dimensionless coupling parameter.

The energy levels $E(g)$ for the Hamiltonian (33) can be represented in the form $E(g)=$ $\left(n+\frac{1}{2}\right) e(g)$, where $n=0,1,2, \ldots$ labels the eigenvalues. The following expansion for function $e(g)$ in powers of the effective coupling:

$$
e_{5}(g)=1+g-\frac{1}{8} g^{2}+\frac{1}{32} g^{3}-\frac{1}{128} g^{4}+\frac{3}{2048} g^{5}
$$

can be found in $[20,40]$. Moreover, for the strong-coupling limit we have

$$
e(g)=\frac{3}{2} g^{2 / 3}+O\left(g^{-2 / 3}\right) \quad(g \rightarrow \infty) .
$$

There are multiple solutions to the minimal sensitivity equation. The best solution to the minimal sensitivity problem corresponds to the complex-conjugate pair

$$
m_{1}^{*}=1.27731+0.320816 i, \quad m_{2}^{*}=1.27731-0.320816 i .
$$

The real part of the corresponding approximants gives the following approximant,

$$
\begin{aligned}
& \prec_{5}^{*}(g)= \\
& 0.5\left(1+(1.27731+0.320816 i) g(1+(0.437208-0.179573 i) g)^{-0.253536+0.0807663 i} \times\right. \\
& \left.(g+1)^{0.110002+0.0795681 i}\right)^{0.736436-0.184967 i}+ \\
& 0.5\left(1+(1.27731-0.320816 i) g(1+(0.437208+0.179573 i) g)^{-0.253536-0.0807663 i} \times\right. \\
& \left.(g+1)^{0.110002-0.0795681 i}\right)^{0.736436+0.184967 i} .
\end{aligned}
$$

Asymptotically, at large $g$

$$
\phi_{5}^{*}(g) \simeq B g^{\beta}
$$

and gives a very good critical index $\beta=0.660389$, and critical amplitude $B=1.50916$.

Compared to all other solutions to the minimal sensitivity condition, Formula (36) gives the smallest (by order of magnitude) error in estimation of the 5 th order coefficient in the expansion.

One can also model Bose-condensate within spherically-symmetrical traps by the following effective Hamiltonian:

$$
\hat{H}_{r}=\frac{1}{2}\left(-\frac{d^{2}}{d r^{2}}+r^{2}\right)+\frac{g}{4 \pi r^{2}} \chi^{2}
$$

for the radial part of the condensate wave function $\chi(r)$ [41].

The ground state energy can be approximated by the expansions

$$
E(c) \simeq \frac{3}{2}+\frac{1}{2} c-\frac{3}{16} c^{2}+\frac{9}{64} c^{3}-\frac{35}{256} c^{4} \quad(c \rightarrow 0),
$$


and

$$
E(c)=\frac{5}{4} c^{2 / 5}+O\left(c^{-2 / 5}\right) \quad(c \rightarrow \infty),
$$

where $c=\frac{g}{(2 \pi)^{3 / 2}}$.

Applying our method, we find $m^{*}=13 / 6 \approx 2.17, \beta_{3} \approx 0.35, B_{3}=1.34$, and reconstruct the large $c$ behaviour

$$
E(c) \simeq B c^{\beta},
$$

with $B=1.34, \beta=0.35$. The third-order coefficient (divided by $a_{0}$ ) could be estimated as well, and is equal to 0.112 , in a reasonable agreement with the $a_{3} / a_{0} \approx 0.14$ from the expansion (38).

Avoiding restrictions on the amplitudes by keeping $\mathcal{A}_{1}(m) \neq \mathcal{A}(m)$, we reconstruct the unrestricted approximation

$$
F_{3}^{*}(x, m)=\left(1+\mathcal{A}(m) x\left(1+\mathcal{A}_{1}(m) x\right)^{s_{1}(m)}\right)^{1 / m} .
$$

and find

$$
\begin{aligned}
& \mathcal{A}(m)=a_{1} m, \\
& \mathcal{A}_{1}(m)=\frac{a_{1}{ }^{4}\left(-m^{2}\right)+6 a_{1}{ }^{4} m-5 a_{1}{ }^{4}-12 a_{1}{ }^{2} a_{2} m+12 a_{1}{ }^{2} a_{2}-24 a_{1} a_{3}+12 a_{2}{ }^{2}}{6 a_{1}\left(a_{1}{ }^{2} m-a_{1}{ }^{2}+2 a_{2}\right)}, \\
& s(m)=-\frac{3\left(a_{1}{ }^{2} m-a_{1}{ }^{2}+2 a_{2}\right)^{2}}{a_{1}{ }^{4} m^{2}-6 a_{1}{ }^{4} m+5 a_{1}{ }^{4}+12 a_{1} a_{2} m-12 a_{1}{ }^{2} a_{2}+24 a_{1} a_{3}-12 a_{2}{ }^{2}} .
\end{aligned}
$$

Optimization condition remains of the same form and brings optimal $m^{*}=6.0512$, with excellent critical index $\beta_{3} \approx 0.39$, and good critical amplitude $B_{3} \approx 1.325$, while the resulting approximant is given as follows,

$$
\phi_{3}^{*}(c)=\frac{3}{2}\left(2.01707 c(0.343481 c+1)^{1.35922}+1\right)^{0.165257} .
$$

The fourth-order coefficient (divided by $a_{0}$ ) could be estimated as well, and is equal to 0.088 , in reasonable agreement with the $a_{4} / a_{0} \approx 0.091$ from the expansion (38). It also reconstructs the large $c$ behaviour

$$
E(c)=B c^{\beta}+O\left(c^{-1}\right),
$$

with the results $B=1.325, \beta=0.39$.

\subsection{Quartic Oscillator}

To conclude our variations on the theme of perturbed harmonic oscillators, let us discuss the very popular quantum model of quartic anharmonic oscillator [42], with the Hamiltonian

$$
\hat{H}=-\frac{1}{2} \frac{d^{2}}{d x^{2}}+\frac{1}{2} x^{2}+g x^{4},
$$

with the anharmonicity parameter $g \in[0, \infty)$.

One can construct the perturbation theory for the ground-state energy in the parameter $g \rightarrow 0$,

$$
e(g) \simeq \frac{1}{2}+\frac{3}{4} g-\frac{21}{8} g^{2}+\frac{333}{16} g^{3}-\frac{30885}{128} g^{4}+\frac{916731}{256} g^{5}-\frac{65518401}{1024} g^{6} .
$$

The expansion in the parameter $g \rightarrow 0$ is divergent also in high orders.

For large $g$ the series for $e(g)$ have fractional powers, with the leading term from the strong-coupling expansion of the energy given as follows,

$$
e(g) \simeq B g^{\beta} \quad(g \rightarrow \infty)
$$

with $\beta=1 / 3, B \approx 0.667986$. 
However, setting $\mathcal{A}_{2}(m)=1$ does not lead to a completely satisfactory solution, although some very reasonable estimates for the critical index and amplitude could be found following the standard calculations, i.e., optimal complex-conjugate pair

$$
m_{1}^{*}=0.0810036-5.35446 i, m_{2}^{*}=0.0810036+5.35446 i,
$$

with excellent critical index $\beta_{5} \approx 0.3213$, and good critical amplitude $B_{5} \approx 0.6979$. However, an overall picture described by a complete approximant $\phi_{5}^{*}(g)$ is not so good, as we were not able to construct an accurate expression for all coupling constants.

However, lifting the restriction on amplitudes, and finding $\mathcal{A}_{2}(m)$ at par with other parameters from the asymptotic equivalence with the truncated weak-coupling expansion, allows to get a consistent expression for all $g$. Using five non-trivial conditions from the weak-coupling expansion and expressing the approximant parameters as functions of $m$, one can find $m$ from the condition of minimal error in predicting the 6th order coefficient, not yet employed. Along this pass we find the following approximant:

$$
\phi_{5}^{*}(g)=\frac{1}{2}\left(1+\frac{16.7519 g(1+1.86188 g)^{2.42764}}{(1+25.4873 g)^{0.0154605}}\right)^{0.0895423} .
$$

with quite reasonable estimates for the critical index $\beta \approx 0.3055$ with error of $8.3 \%$, and the critical amplitude $B \approx 0.7333$. To achieve high accuracy, we still need considerably more terms [22]. The approximant (42) gives good results in pure extrapolation, predicting the ground state energy with the error remaining less than $3 \%$ up to quite large $g=50$, compared with the numerical results [43]. The self-similar approximants from [4] are also rather accurate, giving the critical index close to 0.3 .

The example below shows the value of studying such a model system as the quartic oscillator, as the series to be studied does resemble very much the series for the quartic oscillator.

Let us consider also the energy gap $\delta(z)$ between the lowest and first excited states of the vector boson for the massive Schwinger model in Hamiltonian lattice theory [30,36], which can be represented for strong coupling constants $g$ as follows:

$$
\begin{aligned}
& \delta(z) \simeq 1+2 z-10 z^{2}+ \\
& 78.66667 z^{3}-736.2222 z^{4}+7572.929 z^{5}-82736.69 z^{6}+942803.4 z^{7}
\end{aligned}
$$

where $z=x^{2}, x=\frac{1}{g^{2} a^{2}}$, and $a$ is the lattice spacing. The strong increase of the coefficients makes the series in powers of $z$ widely divergent. They do resemble the truncated expression for the quartic oscillator. The transition from the lattice formulation to the continuous limit requires taking the limit $a \rightarrow 0$, implying $z \rightarrow \infty$. In this limit, the gap behaves as

$$
\delta(z) \sim z^{\beta} \quad(z \rightarrow \infty),
$$

with $\beta=1 / 4$.

There are two good solutions to the optimization problem. The complex-conjugate pair

$$
m_{1}^{*}=7.13701-1.35426 i, m_{2}^{*}=7.13701+1.35426,
$$

with $\beta_{5}=0.22433,(10 \%$ error $)$, and real solution $m^{*}=10.8324$, with $\beta_{5}=0.237578(5 \%$ error). The former solution incurs slightly smaller average error in predicting unexploited three higher-order terms from the expansion (43). Yet, the latter solution is still preferable as it brings a smaller maximal error of $2.7 \%$ in prediction of the remaining three coefficients from the truncation (43). The real solution corresponds to the approximant

$$
\phi_{5}^{*}(z)=\left(1+21.6649 z(1+z)^{0.964953}(1+6.35479 z)^{0.608593}\right)^{0.0923153} .
$$




\section{Finite Critical Point}

Consider another ubiquitous situation when the function $\phi(x)$ of a real variable $x$ exhibits critical behavior,

$$
\phi(x) \simeq B\left(x_{c}-x\right)^{\beta}, \text { as } x \rightarrow x_{c}-0,
$$

with a positive or negative critical index $\beta$, and critical amplitude $B$ at a finite critical point $x_{c}$. The approach developed for the case of a critical point located at infinity can be applied with minor modifications when the critical point $x_{c}$ is finite and its position is known, in conjunction with transformation

$$
z=\frac{x}{x_{c}-x}
$$

while $x=\frac{z x_{c}}{z+1}$. In the low-order case of $k=3$, just like in the case of critical point located at infinity, we obtain explicit and uniquely defined expressions,

$$
\mathcal{A}_{1}(m)=\mathcal{A}(m)=a_{1} m x_{c}, \quad s_{1}(m)=\frac{a_{1}^{2} m x_{c}-a_{1}^{2} x_{c}-2 a_{1}+2 a_{2} x_{c}}{2 a_{1}^{2} m x_{c}} .
$$

The optimization problem remains of the same form, and by solving it we find optimal control parameter

$$
m^{*}=\frac{2}{3}\left(1+\frac{2\left(a_{1}-a_{2} x_{c}\right)}{a_{1}^{2} x_{c}}\right)
$$

It leads to the critical index

$$
\beta \approx \beta_{3}=-\frac{9 a_{1}^{2} x_{c}}{8\left(a_{1}^{2} x_{c}+2 a_{1}-2 a_{2} x_{c}\right)},
$$

and critical amplitude

$$
B \approx B_{3}=\left(\frac{2}{3}\right)^{\frac{9 a_{1}^{2} x_{c}}{8\left(a_{1}^{2} x_{c}+2 a_{1}-2 a_{2} x_{c}\right)}}\left(x_{c}\left(-\frac{2 a_{2} x_{c}}{a_{1}}+a_{1} x_{c}+2\right)\right)^{\frac{9 a_{1}^{2} x_{c}}{8\left(a_{1}^{2} x_{c}+2 a_{1}-2 a 2 x_{c}\right)}} .
$$

A number of examples considered below cover the most interesting for us physical cases. The problems of conductivity, viscosity, and permeability arising in different contexts can be solved based on seemingly sparse information.

\subsection{D Ising Model}

Consider spin-1/2 Ising model characterized by the Hamiltonian

$$
\hat{H}=-\frac{J}{2} \sum_{\langle i j\rangle} s_{i}^{z} s_{j}^{z}
$$

with $s_{j}^{z} \equiv \frac{S_{j}^{z}}{S}$, on a square lattice, with the ferromagnetic interaction $J$ of nearest neighbors, for spins $S_{j}^{z}= \pm 1 / 2$ [44]. The dimensionless interaction parameter is defined as $g \equiv \frac{J}{k_{B} T}$, where $k_{B}$ stands for the Boltzmann constant and $T$ is temperature.

On the square lattice, a high-temperature expansion of the susceptibility $\chi$ in powers of dimensionless inverse temperature $g$ could be obtained in rather high orders [44]. The starting terms of the expansion are given as follows,

$$
\chi(g) \simeq 1+4 g+12 g^{2} .
$$


It is expected [45] that in the vicinity of the threshold $g_{c}=\left(\frac{2}{\log (1+\sqrt{2})}\right)^{-1} \approx 0.440687$, the $2 \mathrm{D}$ susceptibility diverges as

$$
\chi(g) \sim\left(g_{c}-g\right)^{-\gamma}
$$

with exact $\gamma=\frac{7}{4}$.

Application of the formulas presented above gives $m^{*}=0.423062, \gamma=\beta_{3}=-1.77279$, $B_{3}=0.139076$. The critical index is well within the bounds $\gamma=1.76 \pm 0.15$, found in [46]. It is significantly better than our previous result $\gamma=1.923 \pm 0.077$, obtained by applying the technique of root approximants [5].

\subsection{Conductivity of Percolating Systems}

The conductivity for site percolation model is explained as a transport of classical particles through a random medium $[47,48]$. The minimal model of such phenomena is the Lorenz 2D gas, which is realized on a square lattice with a fraction of sites being excluded at random. If $f$ stands for the concentration of conducting or not excluded sites in the Lorenz model, then $x=1-f$ stands for the concentration of excluded sites. Through the diffusion coefficient for random walkers on such a lattice one can express the macroscopic conductivity [47]. The transport ceases to exist at the critical density of the excluded sites $x_{c}$ corresponding to the site percolation threshold [49], and the conductivity behaves as

$$
\sigma(x) \propto\left(x_{c}-x\right)^{t} \quad\left(x \rightarrow x_{c}-0\right),
$$

with $x_{c}=0.4073, t=1.310$. Perturbation theory in powers of the variable $x=1-f$ [47], gives for the two-dimensional square lattice the expansion

$$
\sigma(x) \simeq 1-\pi x+1.28588 x^{2} \quad(x \rightarrow 0) .
$$

In our approach, we obtain $m^{*}=-0.549066, t=\beta_{3}=1.36596, B_{3}=5.52404$. The estimate for the critical index is well within the bounds $t=1.291 \pm 0.1$ found in [46].

The effective conductivity for the three-dimensional site percolation is studied similarly to the two-dimensional one. The conductivity also exhibits the critical behaviour [50-54], described just as in the equation (51), but with $x_{c}=0.688, t=1.9$. Perturbation theory gives

$$
\sigma(x) \simeq 1-2.52 x+1.52 x^{2} \quad(x \rightarrow 0) .
$$

Using our method, we get $m^{*}=-0.421068, t=\beta_{3}=1.78119, B_{3}=3.40252$. The estimate for the critical index is well within the bounds $t=1.82 \pm 0.09$, calculated in [46].

\subsection{Permeability of the Two-Dimensional Channels}

Let us consider the case of a Darcy flow in the two-dimensional channel bounded by the surfaces $z= \pm b(1+\epsilon \cos x)$, where $\epsilon$ is termed waviness. The permeability $K(\epsilon)$ behaves critically $[2,5,55]$. Precisely, it tends to zero as

$$
K(\epsilon) \sim\left(\epsilon_{c}-\epsilon\right)^{\kappa}, \text { as } \epsilon \rightarrow \epsilon_{c}-0,
$$

with $\epsilon_{c}=1, \kappa=\frac{5}{2}$. An expression for permeability can be derived by iterative perturbation method. It gives a truncated expansion in powers of the waviness [2,55-57].

The permeability, for $b=0.5$, has the expansion

$$
K(\epsilon) \simeq 1-3.14963 \epsilon^{2}+4.08109 \epsilon^{4}, \text { as } \epsilon \rightarrow 0,
$$

Application of the formulas presented above gives $m^{*}=-0.305188, \kappa=\beta_{3}=2.4575$, $B_{3}=1.10206$. The critical index is within the bounds $\kappa=2.372 \pm 0.19$ found in [5], but is much closer to the upper bound and to the expected value of $5 / 2$ than to the center. 
The permeability, for $b=0.25$ [56], has the expansion

$$
K(\varepsilon) \simeq 1-3.03748 \varepsilon^{2}+3.54570 \varepsilon^{4}, \text { as } \varepsilon \rightarrow 0 .
$$

Application of the formulas presented above gives $m^{*}=-0.284699, \kappa=\beta_{3}=2.63436$, $B_{3}=0.670918$. The critical index is located well within the bounds $\kappa=2.543 \pm 0.2$ found in [5].

\subsection{Effective Viscosity}

The elasticity problem of perfectly rigid spherical inclusions randomly embedded into an incompressible matrix is analogous to the problem of high-frequency effective viscosity of a hard-sphere suspension [58-60]. The viscosity, considered as a function of the variable $f \equiv \frac{4 \pi}{3} r_{s}^{3} \rho,\left(\rho \equiv \frac{N}{V}\right)$, in which $r_{s}$ is the sphere radius and $\rho$ is average density, exhibits the critical behaviour

$$
\eta(f) \propto\left(f_{c}-f\right)^{-\mathcal{S}} \quad(f \rightarrow f-0),
$$

where $f_{c}=0.637, \mathcal{S}=1.75$ [61]. The small $f$-expansion form [60], reads as

$$
\eta(f) \simeq 1+\frac{5}{2} f+5.0022 f^{2} \quad(f \rightarrow 0) .
$$

Using our method, we find $m^{*}=0.436789, \mathcal{S}=-\beta_{3}=1.71708, B_{3}=0.24717$. The estimate for the critical index appears to fit within the bounds $\mathcal{S}=1.726 \pm 0.06$, obtained in [5]. In what follows, one should distinguish the critical index for viscosity (elasticity) $\mathcal{S}$ from the critical index for superconductivity denoted as s.

\subsection{Critical Index for Superconductivity in Random 3D Case}

In the limiting case of a three-dimensional randomly distributed perfectly conducting inclusions, the effective conductivity $\sigma_{e}$ is expected to tend to infinity as a power law, with critical index s, as the concentration of inclusions $f$ tends to $f_{\mathcal{c}} \approx 0.637$, the maximal value in $3 \mathrm{D}$, and

$$
\sigma_{e}(f) \sim\left(f_{c}-f\right)^{-s}
$$

The superconductivity critical index $s$ is expected to have the value of $0.73 \pm 0.01$ [54]. There is also a slightly larger estimate, $\mathrm{s} \approx 0.76$ [62].

For sample generation, the Random Sequential Adsorption protocol was employed [1]. The consecutive objects were placed randomly in the cell, rejecting those that overlap with the previously absorbed one. For macroscopically isotropic composites, the expansion for scalar effective conductivity was found to be

$$
\sigma_{e}=1+3 f+3 f^{2}+4.80654 f^{3}+O\left(f^{\frac{10}{3}}\right) .
$$

Application of the formulas presented above gives $m^{*}=0.919937, \mathrm{~s}=-\beta_{3}=$ $0.815273, B_{3}=1.09667$.

For the effective conductivity we find the following compact expression

$$
\sigma_{e}(f)=\phi_{3}^{*}(f)=\left(1+\frac{1.758 f}{\left(\frac{0.758 f+0.637}{0.637-f}\right)^{0.25}(0.637-f)}\right)^{1.08703},
$$

which can be expanded in powers of $f$. It does exceptionally well in estimating the coefficient $a_{3} \approx 4.7963$, deviating from the expected value only $0.21 \%$. The 4 th-order coefficient can be estimated as well, $a_{4} \approx 6.68$.

The low-order estimate appears to be self-consistent, because the estimate for the critical index is supported by a simultaneous excellent result for $a_{3}$. Of course, one can attempt to incorporate the third-order coefficient explicitly, i.e., to devise the approximant 
$\mathcal{P}_{3}^{*}(f)$ with $\mathcal{A} \neq \mathcal{A}_{1}$. Computations are still possible to perform in a symbolic form. In such case the estimates become very close to the numerical results of [54], and to the results of calculations with various approximants [2], based on the same number of terms,

$$
\begin{aligned}
& \sigma_{e}(f)=\phi_{3}^{*}(f)= \\
& \frac{1}{2}\left(\left(1-\frac{(2.55836+0.117254 i) f\left(1+\frac{(1.28492-0.744437 i) f}{0.637-f}\right)^{-0.042703+0.0208865 i}}{f-0.637}\right)^{0.745398-0.034163 i}+\right. \\
& \left.\left(1+\frac{(2.55836-0.117254 i) f\left(1-\frac{(1.28492+0.744437 i) f}{f-0.637}\right)^{-0.042703-0.0208865 i}}{0.637-f}\right)^{0.745398+0.034163 i}\right),
\end{aligned}
$$

leading to the critical index $\mathrm{s}=0.714$, and the critical amplitude $B=1.46$. The 4 th order coefficient can be estimated as $a_{4} \approx 6.01$. But to confirm or reject the results one will have to rely on some novel, additional information, which is not available at the moment.

\subsection{Critical Index for Superconductivity of Honeycomb Array}

Finally, consider a regular honeycomb array of perfectly conducting (superconducting) disks. As their volume fraction $f \rightarrow f_{c}$, the effective conductivity of the array goes to infinity as a power law similar to previous example, with critical index s, as the concentration of disks $f$ tends to $f_{c}=\frac{\pi}{3 \sqrt{3}}$.

It is always instructive to study the regular case by yet different methods, other than employed in [63], and estimate the critical index s. The exact value for the index is expected to be $1 / 2$. The small $f$ polynomial has the following form,

$$
\sigma_{e} \simeq 1+2 f+2 f^{2}+2 f^{3}+4.14933 f^{4}+6.29865 f^{5}+8.44798 f^{6} .
$$

There are multiple solutions to the optimization problem understood as solving the minimal sensitivity equation. Note that we have to apply first the transformation $z(f)=\frac{f}{f_{c}-f}$, as in the formula (45), and then construct the critical index $\beta_{5}(m)$ (mind the sign!) and approximant $\phi_{5}^{*}(z(f))$, as shown above in the Section 2.

The best solution to the minimal sensitivity problem corresponds to the complexconjugate pair

$$
m_{1}^{*}=2.46436-0.462709 i, \quad m_{2}^{*}=2.46436+0.462709 i .
$$

The real part of the corresponding approximants gives

$$
\begin{aligned}
& \sigma_{e}(f)=\phi_{5}^{*}(f)= \\
& \frac{1}{2}\left(\left(1+(3.2629+0.850614 i) f\left(1+\frac{(1.56155-0.331863 i) f}{0.6046-f}\right)^{0.375221+0.347239 i} \times\right.\right. \\
& \left.\left(\frac{1}{0.6046-f}\right)^{0.788789-0.137954 i}\right)^{0.391967-0.0735958 i}+ \\
& \left(1+(3.2629-0.850614 i) f\left(\frac{1}{0.6046-f}\right)^{0.788789+0.137954 i} \times\right. \\
& \left.\left.\left(1+\frac{(1.56155+0.331863 i) f}{0.6046-f}\right)^{0.375221-0.347239 i}\right)^{0.391967+0.0735958 i}\right) .
\end{aligned}
$$

Asymptotically, as $f \rightarrow f_{c}$,

$$
\phi_{5}^{*}(g) \simeq B\left(f_{c}-f\right)^{\beta},
$$

with reasonable estimates for critical index $-\beta=\mathrm{s}=0.471655$, and critical amplitude $B=0.862639$. The expected value for the index is $1 / 2$ [63], the same as for other regular arrays of inclusions [1,64].

Consider for reassurance also the toy model represented by the function

$$
\phi(x)=\frac{\sqrt{\frac{1+x}{1-x}}-1}{x}
$$

with transition at the point $x_{c}=1$, index $\beta=1 / 2$ and amplitude $B=\sqrt{2}$. 
As $x \rightarrow 0$

$$
\phi(x) \simeq 1+\frac{x}{2}+\frac{x^{2}}{2}+\frac{3 x^{3}}{8}+\frac{3 x^{4}}{8}+\frac{5 x^{5}}{16}+\frac{5 x^{6}}{16} .
$$

We find that the same conclusions as were reached above for realistic problems, apply here. Namely, there are multiple solutions of the higher-order optimization condition and the best result is achieved for the complex-conjugate pair

$$
m_{1}^{*}=2.89498-1.37644 i, \quad m_{2}^{*}=2.89498+1.37644 i .
$$

The real part of the corresponding approximants gives

$$
\begin{aligned}
& \phi_{5}^{*}(f)=\frac{1}{2} \times \\
& \left(\left(1-\frac{(1.44749+0.688222 i) x\left(\frac{1}{1-x}\right)^{0.720503+0.570413 i}\left(1-\frac{(1.3549+1.04464 i) x}{x-1 .}\right)^{-0.194988-0.0166872 i}}{x-1 .}\right)^{0.281736-0.133954 i}+\right. \\
& \left.\left(1-\frac{(1.44749-0.688222 i) x\left(\frac{1}{1-x}\right)^{0.720503-0.570413 i} \times\left(1-\frac{(1.3549-1.04464 i) x}{x-1}\right)^{-0.194988+0.0166872 i}}{x-1}\right)^{0.281736+0.133954 i}\right),
\end{aligned}
$$

while the critical index $\beta=-0.503966$. As was already suggested above, the good result for the index does not necessarily preclude correspondingly accurate results for the critical amplitude $B \approx 0.451$, as the optimization procedure involves the critical index, but not the critical amplitude. The formula (61) for the effective conductivity appears to be smart, meaning that it does more accurately (by orders of magnitude) than other solutions to the optimization problem, predict the average error for the remaining 2 coefficients from the expansion for conductivity.

\subsection{Compressibility Factor of Hard-Disks Fluids}

The state of hard-disks fluids is described by the compressibility factor

$$
Z=\frac{P}{\rho k_{B} T}=Z(f) \quad\left(f \equiv \frac{\pi \rho}{4} a_{s}^{2}\right)
$$

in which $P$ is pressure, $\rho$ is density, $T$ is temperature, $a_{S}$ is the disk diameter, $k_{B}$ stands for Boltzmann constant, and $f$ is called packing fraction. The compressibility factor exhibits critical behaviour at a finite critical point. This behavior has been found from phenomenological equations as

$$
Z(f) \simeq B\left(f_{c}-f\right)^{\beta} \quad\left(f \rightarrow f_{c}-0\right),
$$

with the fitted parameters $f_{c}=1$ and $\beta=-2[65,66]$, although these are not asymptotically exact values. For low packing fractions the compressibility factor is represented by the virial expansion

$$
\begin{aligned}
& Z(f) \simeq 1+2 f+3.12802 f^{2}+4.25785 f^{3}+5.3369 f^{4}+6.36296 f^{5}+7.35186 f^{6} \\
& +8.3191 f^{7}+9.27215 f^{8}+10.2163 f^{9}
\end{aligned}
$$

from in $[67,68]$. Using the same optimization methods as above, we find $m^{*}=0.808791$, $\beta_{5}=-1.80539, B_{5}=1.84906$ and the approximant

$$
\phi_{5}^{*}(f)=\left(1+\frac{1.61758\left(\frac{1}{1-f}\right)^{0.831449} f}{(1-f)\left(1+\frac{1.23536 f}{1-f}\right)^{0.371267}}\right)^{1.23641}
$$

which employs the terms up to the 4 th order in $f$ and predicts the rest with maximal error of just $0.52 \%$. Simply setting $m^{*}=1$ leads to a simpler expression

$$
\phi_{5}^{*}(f, 1)=1+\frac{2 f\left(\frac{1-0.122783 f}{1-f}\right)^{2.29987}}{\left(\frac{1}{1-f}\right)^{0.453479}},
$$


which works with almost the same accuracy in predicting the coefficients from the truncated series (64). The latter formula may yet have advantage in predicting the higher-order coefficients. Such coefficients were only estimated up to 15th order (see, e.g., the book [2] and references therein), but are not exact unlike the others.

\section{Concluding Remarks}

We conclude that "odd" factor approximants of the special form represented by the Formula (11) are amenable to optimization by power transformation and can be successfully applied to the critical phenomena of various physical nature.

The novelty of the current approach amounts to the idea that the critical index by itself should be optimized through the parameters of power transform calculated from the optimization condition. The critical index is a product of the algebraic transformation which contributes to the expressions the set of control parameters $s_{i}$, and of the power transform which corrects them.

The parameter of power transformation $m$ has the simple meaning of the multiplier connecting the critical exponent with the correction-to-scaling exponent. Both, the power transform and minimal sensitivity conditions are of a non-perturbative nature. As we optimize the critical index directly the results for the indices appear to be more accurate than obtained from optimization of critical amplitudes with a subsequent determination of the indices with optimal conditions calculated for the amplitudes.

We study mostly the minimal model of critical phenomena based on expansions with only two coefficients and critical points. The power-transformed factor approximants of the form (11) are asymptotically equivalent to such series. The approximants are optimized by complementing them with natural optimization conditions. The minimal sensitivity condition imposed on the critical index appears to bring quite accurate, uniquely defined results given by simple formulas. Surprisingly, many important cases of critical phenomena are being covered by the simple formulae. The knowledge of higher-order coefficients appears to be excessive as critical indices could be estimated from only two low-order coefficients. The multitude of the unknown higher-order coefficients can be mimicked by optimized power-transformed factor approximations.

For the longer series, the optimization condition possesses multiple solutions and additional constraints should be applied. In particular we require that the chosen solution is to be best in prediction of the coefficients $a_{k}$ not employed in its construction. In principle, the error/measure of such prediction can be optimized by itself, with respect to the parameter of power transform. The latter idea dwells on the requirement of independence of the critical indices from the higher-order coefficients $a_{n}$. Some other approaches to long series were discussed in the preceding work $[2,22]$.

Methods of calculation based on optimized power-transformed factors are applied and results presented for critical indices of several key models of conductivity and viscosity of random media, swelling of polymers, and permeability in two-dimensional channels. Several quantum mechanical problems based on the strongly perturbed harmonic oscillator are discussed as well.

Accurate calculations with short truncated series are possible and accurate in quite a few important cases, because the higher-order coefficients appear to be redundant close to the critical point, and critical indices could be estimated just from two low-order coefficients by imposing some universal conditions of non-perturbative nature. Power transform extends the class of approximations and brings some unique quality, such as incorporating both critical and sub-critical indices simultaneously. The optimization procedure is developed to estimate the indices together.

Convergence is not always as fast as in the examples presented above. To improve results one would try adding more terms from the truncation when possible, and apply different optimized approximations, or even introducing control functions instead of control parameters [1,22]. On the other hand, the methodology is limited to a short series because the optimization procedure developed above is based on analytical expressions 
for the parameters of approximants. Such limitation could be overcome by resorting to different self-similar approximants of the type described in [20].

One can think (see, e.g., in [18]) that for an approach based on optimization to be successful, Nature by itself should be organized in such a way that certain quantities called critical indices play a special role of stabilizing various physical phenomena in the vicinity of their respective critical points by making them minimally sensitive to the parameters of power transform.

Critical indices are introduced into consideration by assuming a trial power law. They could be found from special optimization conditions of general nature selecting a unique fixed point/sum of the asymptotic series associated with a given truncation. Such fixed points also incorporate the critical behavior which appears to be optimal.

Funding: This research received no external funding.

Informed Consent Statement: Not applicable.

Conflicts of Interest: The author declares no conflict of interest.

\section{References}

1. Gluzman, S.; Mityushev, V.; Nawalaniec, W. Computational Analysis of Structured Media; Academic Press: Cambridge, MA, USA, 2017.

2. Drygaś, P.; Gluzman, S.; Mityushev, V.; Nawalaniec, W. Applied Analysis of Composite Media; Woodhead Publishing: Sawston, UK, 2020.

3. Gluzman, S.; Yukalov, V.I. Self-Similar Power Transforms in Extrapolation Problems. J. Math. Chem. 2006, 39, 47-56. [CrossRef]

4. Gluzman, S.; Yukalov, V.I. Extrapolation of perturbation theory expansions by self-similar approximants. Eur. J. Appl. Math. 2014, 25, 595-628. [CrossRef]

5. Gluzman, S.; Yukalov, V.I. Critical indices from self-similar root approximants. Eur. Phys. J. Plus 2017, 132, 535. [CrossRef]

6. Gluzman, S. Optimized Factor Approximants and Critical Index. Symmetry 2021, 13, 903. [CrossRef]

7. Yukalov, V.I.; Gluzman, S. Optimization of Self-Similar Factor Approximants. Mol. Phys. 2009, 107, 2237-2244. [CrossRef]

8. Tukey, J.W. Exploratory Data Analysis; Addison-Wesley: Reading, MA, USA, 1977.

9. Box, G.E.; Cox, D.R. An analysis of transformations. J. R. Stat. Soc. Ser. B 1964, 26, 211-252. [CrossRef]

10. Carlson, J.M.; Doyle, J. HOT: A mechanism for power laws in designed systems. Phys. Rev. E 1999, 60, 1412-1427. [CrossRef] [PubMed]

11. Yukalov, V.I. Theory of perturbations with a strong interaction. Mosc. Univ. Phys. Bull. 1976, 51, 10-15.

12. Yukalov, V.I. Model of a hybrid crystal. Theor. Math. Phys. 1976, 28, 652-660. [CrossRef]

13. Kadanoff, L.P.; Houghton, A. Numerical evaluations of the critical properties of the two-dimensional Ising model. Phys. Rev. B 1975, 11, 377-386. [CrossRef]

14. Stevenson, P.M. The effective exponent $\gamma(Q)$ and the slope of the $\beta$-function. Phys. Lett. B 2016, 761, 428-430. [CrossRef]

15. Kleinert, H. Path Integrals in Quantum Mechanics, Statistics, Polymer Physics and Financial Markets; World Scientific: Singapore, 2006.

16. Suzuki, M. Statistical Mechanical Theory of Cooperative Phenomena.I. General Theory of Fluctuations, Coherent Anomalies and Scaling Exponents with Simple Applications to Critical Phenomena. J. Phys. Soc. Jpn. 1986, 55, 4205-4230. [CrossRef]

17. Suzuki, M. Continued-Fraction CAM Theory. J. Phys. Soc. Jpn. 1988, 57, 1-4. [CrossRef]

18. Yukalov, V.I.; Gluzman, S. Critical Indices as Limits of Control Functions. Phys. Rev. Lett. 1997, 79, 333-336. [CrossRef]

19. Gluzman, S.; Yukalov, V.I. Additive self-similar approximants. J. Math. Chem. 2017, 55, 607-622. [CrossRef]

20. Gluzman, S.; Yukalov, V.I. Self-similar continued root approximants. Phys. Lett. 2012, 377, 124-128. [CrossRef]

21. Gluzman, S.; Yukalov, V.I. Algebraic self-similar renormalization in theory of critical phenomena. Phys. Rev. E 1997, 55, 3983-3999. [CrossRef]

22. Gluzman, S. Padé and post-Padé approximations for critical phenomena. Symmetry 2020, 12, 1600. [CrossRef]

23. Muthukumar, M.; Nickel, B.G. Perturbation theory for a polymer chain with excluded volume interaction. J. Chem. Phys. 1984, 80, 5839-5850. [CrossRef]

24. Muthukumar, M.; Nickel, B.G. Expansion of a polymer chain with excluded volume interaction. J. Chem. Phys. 1987, 86, 460-476. [CrossRef]

25. Grosberg, A.Y.; Khokhlov, A.R. Statistical Physics of Macromolecules; AIP Press: Woodbury, NY, USA, 1994.

26. Pelissetto, A.; Vicari, E. Critical phenomena and renormalization-group theory. Phys. Rep. 2002, 368, 549-727. [CrossRef]

27. Li, B.; Madras, N.; Sokal, A.D. Critical exponents, hyperscaling, and universal amplitude ratios for two- and three-dimensional self-avoiding walks. J. Stat. Phys. 1995, 80, 661-754. [CrossRef]

28. Clisby, N. Accurate estimate of the critical exponent for self-avoiding walks via a fast implementation of the pivot algorithm. Phys. Rev. Lett. 2010, 104, 055702. [CrossRef] 
29. Caracciolo, S.; Guttmann, A.J.; Jensen, I.; Pelissetto, A.; Rogers, A.N.; Sokal, A.D. Correction-to-scaling exponents for twodimensional self-avoiding walks. J. Stat. Phys. 2005, 120, 1037-1100. [CrossRef]

30. Schwinger, J. Gauge invariance and mass. Phys. Rev. 1962, 128, 2425-2428. [CrossRef]

31. Banks, T.; Susskind, L.; Kogut, J. Strong-coupling calculations of lattice gauge theories: $(1+1)$-dimensional exercises. Phys. Rev. D 1976, 13, 1043-1053. [CrossRef]

32. Carrol, A.; Kogut, J.; Sinclair, D.K.; Susskind, L. Lattice gauge theory calculations in $1+1$ dimensions and the approach to the continuum limit. Phys. Rev. D 1976, 13, 2270-2277. [CrossRef]

33. Vary, J.P.; Fields, T.J.; Pirner, H.J. Chiral perturbation theory in the Schwinger model. Phys. Rev. D 1996, 53, 7231-7238. [CrossRef] [PubMed]

34. Adam, C. The Schwinger mass in the massive Schwinger model. Phys. Lett. B 1996, 382, 383-388. [CrossRef]

35. Striganesh, P.; Hamer, C.J.; Bursill, R.J. A new finite-lattice study of the massive Schwinger model. Phys. Rev. D 2000, 62, 034508. [CrossRef]

36. Hamer, C.J.; Weihong, Z.; Oitmaa, J. Series expansions for the massive Schwinger model in Hamiltonian lattice theory. Phys. Rev. D 1997, 56, 55-67. [CrossRef]

37. Coleman, S. More about the massive Schwinger model. Ann. Phys. 1987, 101, 239-267. [CrossRef]

38. Hamer, C.J. Lattice model calculations for SU(2) Yang-Mills theory in $1+1$ dimensions. Nucl. Phys. B 1977, 121, 159-175. [CrossRef]

39. Cioslowski, J. Robust interpolation between weak-and strong-correlation regimes of quantum systems. J. Chem. Phys. 2012, 136, 044109. [CrossRef]

40. Yukalov, V.I.; Yukalova, E.P.; Gluzman, S. Self-similar interpolation in quantum mechanics. Phys. Rev. A 1998, 58, 96-115. [CrossRef]

41. Courteille, P.W.; Bagnato, V.S.; Yukalov, V.I. Bose-Einstein Condensation of Trapped Atomic Gases. Laser Phys. 2001, 11, 659-800.

42. Bender, C.M.; Wu, T.T. Anharmonic oscillator. Phys. Rev. 1969, 184, 1231-1260. [CrossRef]

43. Hioe, F.T.; McMillen, D.; Montroll, E.W. Quantum theory of anharmonic oscillators: energy levels of a single and a pair of coupled oscillators with quartic coupling. Phys. Rep. 1978, 43, 305-335. [CrossRef]

44. Butera, P.; Comi, M. A library of extended high-temperature expansions of basic observables for the spin-S Ising models on twoand three-dimensional lattices. J. Stat. Phys. 2002, 109, 311-315. [CrossRef]

45. Baxter, R.J. Exactly Solved Models in Statistical Mechanics; Academic: London, UK, 1982.

46. Gluzman, S. Critical Index for Conductivity, Elasticity, Superconductivity. Results and Methods. In Mechanics and Physics of Structured Media; Andrianov, I., Gluzman, S., Mityushev, V., Eds.; Elsevier: Amsterdam, The Netherlands, 2021.

47. Nieuwenhuizen, T.M.; van Velthoven, P.F.J.; Ernst, M.H. Diffusion and long-time tails in a two-dimensional site-percolation model. Phys. Rev. Lett. 1986, 57, 2477-2480. [CrossRef]

48. Frenkel, D. Velocity auto-correlation functions in a 2d lattice Lorentz gas: Comparison of theory and computer simulation. Phys. Lett. 1987, 121, 385-389. [CrossRef]

49. Grassberger, P. Conductivity exponent and backbone dimension in 2d percolation. Phys. A 1999, 262, 251-263. [CrossRef]

50. Ziff, R.M.; Torquato, S. Percolation of disordered jammed sphere packings. J. Phys. A Math. Theor. 2017, 50, 085001. [CrossRef]

51. Kirkpatrick, S. Percolation and Conduction. Rev. Mod. Phys. 1973, 45, 574-588. [CrossRef]

52. Hofling, F.; Franosch, T.; Frey, E. Localization transition of the three-dimensional Lorenz model and continuum percolation. Phys. Rev. Lett. 2006, 96, 165901. [CrossRef]

53. Bauer, T.; Hofling, F.; Munk, T.; Frey, E.; Franosch, T. The localization transition of the two-dimensional Lorenz model. Eur. Phys. J. Spec. Top. 2010, 189, 103-118. [CrossRef]

54. Clerc, J.P.; Giraud, G.; Laugie, J.M.; Luck, J.M. The electrical conductivity of binary disordered systems, percolation clusters, fractals and related models. Adv. Phys. 1990, 39, 191-309. [CrossRef]

55. Adler, P.M. Porous Media. Geometry and Transport; Butterworth-Heinemann: New York, NY, USA, 1992.

56. Malevich, A.E.; Mityushev, V.V.; Adler, P.M. Stokes flow through a channel with wavy walls. Acta Mech. 2006, 182, 151-182. [CrossRef]

57. Gluzman, S. Nonlinear approximations to critical and relaxation processes. Axioms 2020, 9, 126. [CrossRef]

58. Batchelor, G.K.; Green, J.T. The determination of the bulk stress in a suspension of spherical to order $c^{2}$. J. Fluid Mech. 1972, 56, 401-427. [CrossRef]

59. Brady, J.F. The rheological behavior of concentrated colloidal dispersions. J. Chem. Phys. 1993, 99, 567-581. [CrossRef]

60. Wajnryb, E.; Dahler, J.S. The Newtonian viscosity of a moderately dense suspensions. Adv. Chem. Phys. 1997, 102, 193-314.

61. Losert, W.; Bocquet, L.; Lubensky, T.C.; Gollub, J.P. Particle dynamics in sheared granular matter. Phys. Rev. Lett. 2000, 85, 1428-1431. [CrossRef]

62. Bergman, D.J.; Stroud, D. Physical properties of macroscopically inhomogeneous media. Solid State Phys. 1992, 46, 148-270.

63. Drygaś, P.; Filshtinski, L.A.; Gluzman, S.; Mityushev,V. Conductivity and elasticity of graphene-type composites. In 2D and Quasi-2D Composite and Nano Composite Materials, Properties and Photonic Applications; McPhedran, R., Gluzman, S., Mityushev, V., Rylko, N., Eds.; Elsevier: Amsterdam, The Netherlands, 2020; Chapter 8, pp. 193-231.

64. Perrins, W.T.; McKenzie, D.R.; McPhedran, R.C. Transport properties of regular array of cylinders. Proc. R. Soc. A 1979, 369, 207-225.

65. Mulero, A.; Cachadina, I.; Solana, J.R. The equation of state of the hard-disc fluid revisited. Mol. Phys. 2009, 107, 1457-1465. [CrossRef] 
66. Santos, A.; Lopez de Haro, M.; Bravo Yuste, S. An accurate and simple equation of state for hard disks. J. Chem. Phys. 1995, 103, 4622-4625. [CrossRef]

67. Clisby, N.; McCoy, B.M. Ninth and tenth order virial coefficients for hard spheres in D dimensions. J. Stat. Phys. 2006, 122, 15-57. [CrossRef]

68. Maestre, M.A.G.; Santos,A.; Robles, M.; de Haro, M.L. On the relation between virial coefficients and the close-packing of hard disks and hard spheres. J. Chem. Phys. 2011, 134, 084502. [CrossRef] [PubMed] 